\title{
Developers Guide for Android Applications
}

\author{
Chaitra M N*, Khateeja Ambareen'S. Meenakshi Sundaram \\ Dept. of Computer Science and Engineering, GSSS Institute of Engineering and Technology for \\ Women, Mysuru.
}

DOI: https://doi.org/10.21467/proceedings.1.36

* Corresponding author email: chaitramn.sep21@gmail.com

\begin{abstract}
Android is most popular OS for smart phones, tablets and it holds almost $79 \%$ market share in global device market. Everyone having website wants to have equivalent smartphone (Android, iOS, Windows phone etc.) apps because more and more people are using smart phone and tablets these days. Having smart phones apps for business is necessary so that they can reach to large audience. This paper concentrates on the survey that I've covered for web applications and android app, what are the features and the tools required to build an android application.
\end{abstract}

Index Terms- Smartphone, IOS, Android, Mobile Operating system.

\section{INTRODUCTION}

Android is a mobile operating system that is based on a modified version of Linux. It was originally developed by a startup of the same name, Android, Inc. In 2005, as part of its strategy to enter the mobile space, Google purchased Android and took over its development work (as well as its development team). [1]

The main advantage of adopting Android is that it offers a unified approach to application development. Developers need only develop for Android, and their applications should be able to run on numerous different devices, as long as the devices are powered using Android. In the world of smartphones, applications are the most important part of the success chain. Device manufacturers therefore see Android as their best hope to challenge the onslaught of the iPhone, which already commands a large base of applications. [2]

\subsection{FEATURES OF ANDROID}

Android is a powerful operating system competing with Apple 4GS and supports great features. Few of them are listed below

1. Beautiful UI - Android OS basic screen provides a beautiful and intuitive user interface.

2. Connectivity - GSM/EDGE, IDEN, CDMA, EV-DO, UMTS, Bluetooth, Wi-Fi, LTE, NFC and WiMAX.

3. Storage - SQLite, a lightweight relational database, is used for data storage purposes.

4. Media support - H.263, H.264, MPEG-4 SP, AMR, AMR-WB, AAC, HE-AAC, AAC 5.1, MP3, MIDI, Ogg Vorbis, WAV, JPEG, PNG, GIF, and BMP.

(C) 2018 Copyright held by the author(s). Published by AIJR Publisher in Proceedings of the $3^{\text {rd }}$ National Conference on Image Processing, Computing, Communication, Networking and Data Analytics (NCICCNDA 2018), April 28, 2018.

This is an open access article under Creative Commons Attribution-NonCommercial 4.0 International (CC BY-NC 4.0) license, which permits any non-commercial use, distribution, adaptation, and reproduction in any medium, as long as the original work is properly cited. ISBN: $978-81-936820-0-5$ 
5. Web browser - Based on the open-source WebKit layout engine, coupled with Chrome's V8 JavaScript engine supporting HTML5 and CSS3.

6. Multi-touch - Android has native support for multi-touch which was initially made available in handsets such as the HTC Hero.

7. Multi-tasking - User can jump from one task to another and same time various application can run simultaneously.

8. Resizable widgets - Widgets are resizable, so users can expand them to show more content or shrink them to save space.

9. Wi-Fi Direct - A technology that lets apps discover and pair directly, over a highbandwidth peer-to-peer connection.

10. Android Beam - A popular NFC-based technology that lets users instantly share, just by touching two NFC-enabled phones together.

11. GCM - Google Cloud Messaging (GCM) is a service that lets developers send short message data to their users on Android devices, without needing a proprietary sync solution. [3]
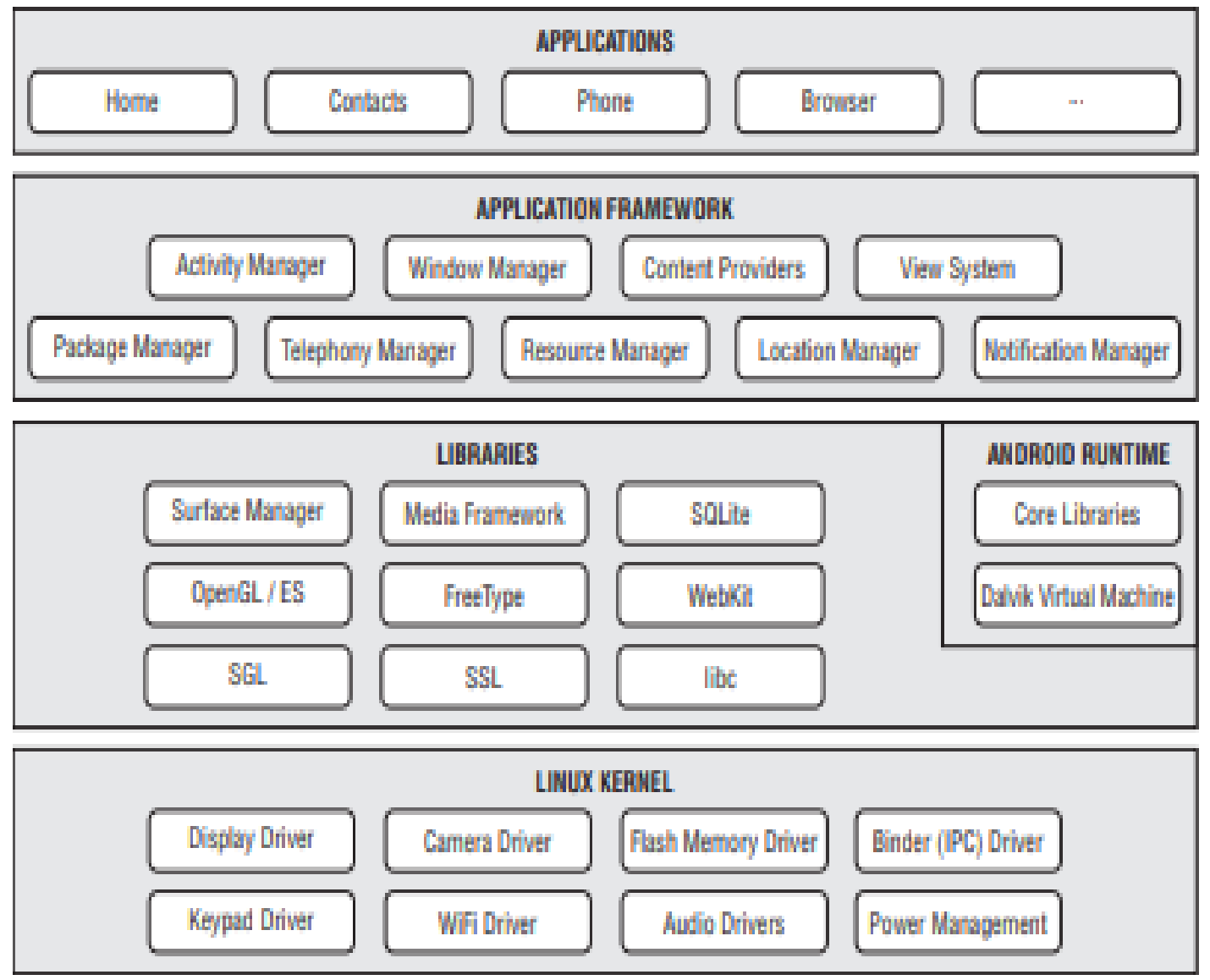

Fig 1.1 Layers of Android Os

Proceedings of the $3^{\text {rd }}$ National Conference on Image Processing, Computing, Communication, Networking and Data Analytics (NCICCNDA 2018) 
Developers Guide for Android Applications

\section{LITERATURE SURVEY}

Constant demand for mobile applications and relatively easy access to the market has been drawing developers into mobile application development. Derivco, a game development company initiated a website project E-Sports Portal. In this project, a mobile application is needed to be developed. Before jumping into developing, background research should be done in the area of software engineering, different platforms and design principles. This literature review aims to cover these areas in general and provide a starting point in developing mobile application. [4] Open handset Alliance focuses on development of Android applications to be used on a handheld device running the Android Operating System (OS). It covers the basics such as setting up a development environment, downloading appropriate tools and add-ons. This paper serves as a guideline to intermediate developers, seeking solutions to problems not discussed in available textbooks. Android is a platform consisting of an operating system and a Software Development Kit (SDK) for handheld devices. It originates from a small software company, acquired by Google and is now owned by Open handset Alliance (OHA), where Google is a member. A description of Android is included, and its advantages and disadvantages are discussed. Resources and recommendations on Android development and Android versioning are presented. Strategies on development are also suggested throughout this paper. The development for an Android device and its data traffic characteristics is of interest, which is also included in this paper. Connectivity and communication like connecting to face book and pulling data from an internet-connected web server, is discussed. [5] Development techniques for android platform mobile device application focuses on Android application development techniques needed to implement a mobile application portal that consists of features used at Eastern Michigan University. Since there is not a single source available to developers that explains such techniques, it represents a unique manual for such development. Based on the similarity of features, mainly in terms of data nature and access, five techniques are defined in a step-by-step procedural manner. This is accomplished by outlining the development techniques and presenting them "in action" with coding examples from a fully developed demo application. As a result, the demo application demonstrates functional solutions to research problems that are able to operate on an actual Android device. And also it provides a unique approach to Android development due to its single focus on the data and IT environment. [6] Android application development is to investigate the design and implementation process of an Android application which uses a web service. The idea of the application was to join an Android user and an Android developer for creating better applications. In addition, the objective of this paper was to develop a prototype which operates with the Parse web service. The theoretical part discusses the Android platform, its history, the compatible web services and the methods used. The empirical part describes the whole process of developing an Android application which uses a web service. The emphasis was set on finding out how a program would work with the Parse web service. The tools that were used during the development phase were Eclipse with the ADT plug-in, the Android SDK 
and the Parse SDK. The result is working Android application which can connect to the Parse web service. The application can input and retrieve data from it and display information to the user. Android application development concludes that the study at hand can be extended with further development of the application by improving the design and adding more functionality. Moreover, it is possible to improve the user experience by creating a website for the application which would use the same web service.

\section{Android Applications}

Android applications are usually developed in the Java language using the Android Software Development Kit. Once developed, Android applications can be packaged easily and sold out either through a store such as Google Play or the Amazon Appstore. Android powers hundreds of millions of mobile devices in more than 190 countries around the world. It's the largest installed base of any mobile platform and is growing fast. Every day more than 1 million new Android devices are activated worldwide. It will start from environment setup for Android application programming and then drill down to look into various aspects of Android applications

\section{Tools required for developing an android application}

\subsection{Setup Java Development Kit (JDK)}

Download the latest version of Java JDK from Oracle's Java site: Java SE Downloads. For installing JDK in downloaded files, follow the given instructions to install and configure the setup. Finally, set PATH and JAVA_HOME environment variables to refer to the directory that contains java and javac, typically java_install_dir/bin and java_install_dir respectively.

\subsection{Setup Android SDK}

Download the latest version of Android SDK from Android's official website: http://developer.android.com/sdk/index.html. For installing SDK on Windows machine, then find ainstaller_rXX-windows.exe, so just download and run this exe which will launch Android SDK Tool Setup wizard to guide you throughout the installation, so just follow the instructions carefully. Finally, Android SDK Tools installed on the machine.

If installing SDK either on Mac OS or Linux, check the instructions provided along with the downloaded android-sdk_rXX-macosx.zip file for Mac OS and android-sdk_rXX-linux.tgz file for Linux. So, launch Android SDK Manager using the option All Programs > Android SDK Tools > SDK Manager.

\subsection{Setup Eclipse IDE}

To install Eclipse IDE, download the latest Eclipse binaries from http://www.eclipse.org/downloads/. Once you have downloaded the installation, unpack the binary distribution into a convenient location. For example, in C: \eclipse on windows, or /usr/local/eclipse on Linux and finally set PATH variable appropriately.

Proceedings of the $3^{\text {rd }}$ National Conference on Image Processing, Computing, Communication, Networking and Data Analytics (NCICCNDA 2018) 
Developers Guide for Android Applications

\subsection{Setup Android Development Tools (ADT) Plugin}

This step will help you in setting Android Development Tool plugin for Eclipse. Start with launching Eclipse and then, choose Help > Software Updates > Install New Software.

Use Add button to add ADT Plugin as name and https://dl-ssl.google.com/android/eclipse/ as the location. Then click OK to add this location. As soon as you will click OK button to add this location, Eclipse starts searching for the plug-in available in the given location and finally lists down the found plugins.

\subsection{Create Android Virtual Device}

The requirement to test Android applications is a virtual Android device. Initially Create an Android virtual device. Launch Android AVD Manager using Eclipse Menu options Window $>$ AVD Manager> which will launch Android AVD Manager. Use New button to create a new Android Virtual Device and enter the following information, before clicking Create AVD button. If AVD is created successfully it means the environment is ready for Android application development. Better to re-start your machine and once done with this last step. [1]

\section{CONCLUSION}

This paper was surveyed on the web applications and android applications. The purpose was to survey and build an android application and a web application. There by, this paper was concentrated on the architecture of an android application, how it has to be built and different layers that had to be included, the different tools that were required to build an android application and the features that had to be overviewed and taking all the features into consideration, the web application had to be developed.

\section{References}

[1] Android tutorial, "Developers guide for android application tutorial". Android tutorial[online]. Available: https://www.tutorialspoint.com/android/android_tutorial.pdf

[2] Android Book, "Android basics". Android book [online]. Available: http://www.kmvportal.co.in/Course/MAP/Android\%20Book.pdf.

[3] Programming android app development tutorials, "Programming android app development tutorials". Android book [online]. Available: http://www.technonutty.com/2015//04/programming-android-app-developmenttutorials-pdf.html

[4] Literature review, "Literature review". Literature review [online]. Available: http://people.cs.uct.ac.za/ cpatrick/Honours/files/LiteratureReviewNHXHAY001.pdf.

[5] Android Application development, "Android app development". Android app [online]. Available: http://muep.mau.se/bitstream/handle/2043/10721/AndroidApplicationDevelopment.pdf?sequence=1.

[6] Android theses, "Android theses". Android book [online]. Available: http://commons.emich.edu/cgi/viewcontent.cgi?article=1762\&context=theses 